\title{
Do Heterogeneous Sediment Properties and Turbulent Velocity Fluctuations have Something in Common? Some History and a New Stochastic Process
}

\begin{abstract}
Fred J. Molz ${ }^{1}$, Mark M. Meerschaert ${ }^{2}$, Tom J. Kozubowski ${ }^{3}$, and Paul D. Hyden ${ }^{4}$
It is increasingly apparent that sediment property distributions on sufficiently small scales are probably irregular. This has led to the development of stochastic theory in subsurface hydrology, including statistically heterogeneous concepts based mainly on the Gaussian and Lévy-stable probability density functions (PDFs), the mathematical basis for stochastic fractals. Gaussian and Levy-stable stochastic fractals have been applied both in the field of turbulence and subsurface hydrology. However, measurements have shown that the increment frequency distributions do not always follow Gaussian or Lévy-stable PDFs. Provided herein is an overview of the origin and development of a new non-stationary stochastic process, called fractional Laplace motion (flam) with stationary, correlated, increments called fractional Laplace noise (fLan). It is based on the Laplace PDF and known generalizations, and does not display self-similarity. Uncorrelated versions are equivalent to a Brownian motion subordinated to the gamma process. In analogy to the development of fractional Brownian motion ( $\mathrm{fBm}$ ) from Brownian motion, fLam is equivalent to $\mathrm{fBm}$ subordinated to a gamma process. The new stochastic fractal has increment PDFs that compare better with measurements, the moments of the PDF family remain bounded, and decay of the increment distribution tails vary from being slower than exponential through exponential and on to a Gaussian decay as the lag size increases. This leads to increasingly more intermittent fluctuations as the lag size decreases. It may be that the geometric central limit theorem, and possible generalizations, will play an important role in connecting the abstract mathematics to the physics underlying applications.
\end{abstract}

\section{HISTORICAL OVERVIEW}

${ }^{1}$ Environmental Engineering and Geology, Clemson University, Anderson, South Carolina.

${ }^{2}$ Mathematics and Statistics, University of Otago, Dunedin, New Zealand.

${ }^{3}$ Mathematics and Statistics, University of Nevada, Reno, Nevada. ${ }^{4}$ Mathematical Sciences, Clemson University, Clemson, South Carolina.

xxxxxxxx Book Title xxxxxxxxxxxxxxxx

Geophysical Monograph Series xxx

Copyright 2005 by the American Geophysical Union

10.1029/xxxGM04

\subsection{Development of Theory}

During the past 3 decades, it has become increasingly apparent that heterogeneity in natural sediments is pervasive, with the implication that property distributions on sufficiently small scales are probably irregular. (Within this chapter, the term "irregular" will mean continuous functions with discontinuous first derivatives or discontinuous functions.) As this concept developed, various individuals were motivated to characterize heterogeneous property distributions, such as $\ln (\mathrm{K}) ; \mathrm{K}=$ hydraulic conductivity, using stochastic concepts. In subsurface hydrology, a significant step forward was taken by Freeze [1975], who began to apply time-series 


\section{HETEROGENEOUS SEDIMENT PROPERTIES AND TURBULENCE}

concepts to $\mathrm{K}$ distributions, with time replaced by distance. He considered $\ln (\mathrm{K})$ to be normally distributed in space, with the mean and variance independent of position. This is an example of what we now call a spatially stationary, uncorrelated Gaussian process. This was later followed by stationary correlated Gaussian processes [Gelhar and Axness, 1983], which served as the basis for a great deal of research during the 1980s and 1990s. This body of work may now be viewed as classical stochastic subsurface hydrology, which is applicable to statistically homogeneous (spatially stationary) porous media.

In order for the statistical parameters (mean, variance, etc.) of a stochastic process to be well-defined, it is necessary that the process be stationary. In the present context, this means that the parameters are not a function of position, as illustrated in Figure 1. Examination of many data sets, however, such as those shown in Figure 2, have indicated that in general heterogeneous $\mathrm{K}$ or $\ln (\mathrm{K})$ distributions are not stationary, meaning that different portions of the property distribution behave very differently in a statistical sense. For example, the distribution plotted in Figure 2 (Transect X) appears to be composed of three distinct portions - an initial moderately variable portion, a central slightly variable portion, and a third highly variable portion. Thus, mean and variance changes with position along the transect where the property was measured [Castle et al., 2004]. A distribution such as this may be called statistically heterogeneous rather than statistically homogeneous. It appears that most property distributions in nature fall in the statistically heterogeneous category [Molz et al., 2004].

Attempting to deal with non-stationary property distributions leads one to consider the subject area of non-stationary stochastic processes with stationary increments. In this approach it is accepted that the property of interest, say $\ln (\mathrm{k})$ ( $\mathrm{k}=$ intrinsic permeability), is non-stationary (statistically heterogeneous), but it is hypothesized that the increments or fluctuations of the property distribution constitute a stationary, and therefore well-defined, stochastic process. In fact, the increments of many $\ln (\mathrm{k})$ data sets appear to be stationary. Such a situation is shown in Figure 3, where the increment data from Figure 2, along with additional data from the same site [Castle et al., 2004], are plotted as a function of along-bed distance. The increment plot is a good approximation of a stationary process, and this motivates the exploration and study of the statistical properties of increment distributions. In the field of turbulence, stationary velocity increment distributions
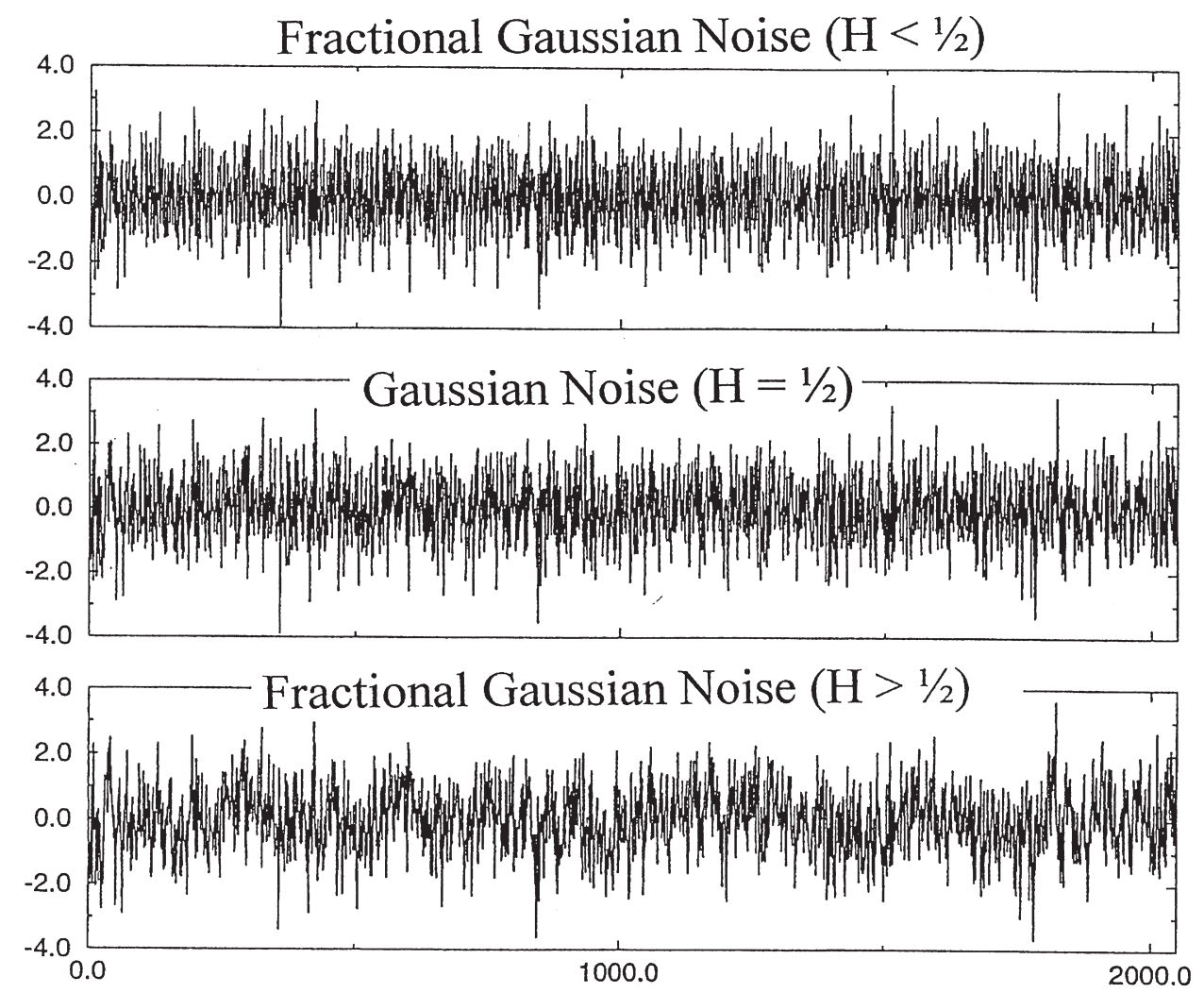

Figure 1. Realization of fractional Gaussian noise (Hurst Coefficient H) for Log K increments. Each plot represents a stationary stochastic process. 

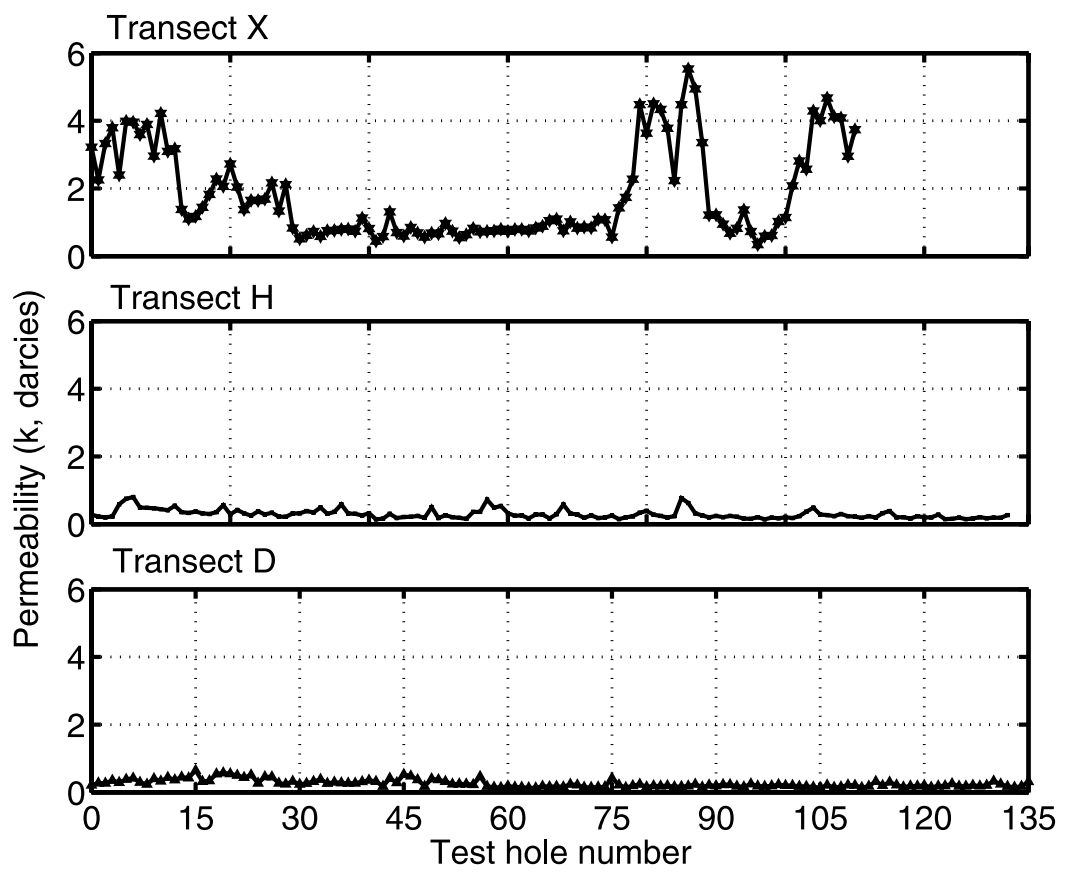

Figure 2. Plots of permeability (k) in millidarcys (vertical coordinates) along three horizontal transects in a near-shore sandstone. The data were obtained using the small-drill-hole gas mini-permeameter [Castle et al., 2004]. The test holes are separated by $15 \mathrm{~cm}$, and the three transects are spread vertically over about $1.5 \mathrm{~m}$. In a statistical sense, the plots display non-stationary behavior in both the vertical and horizontal.

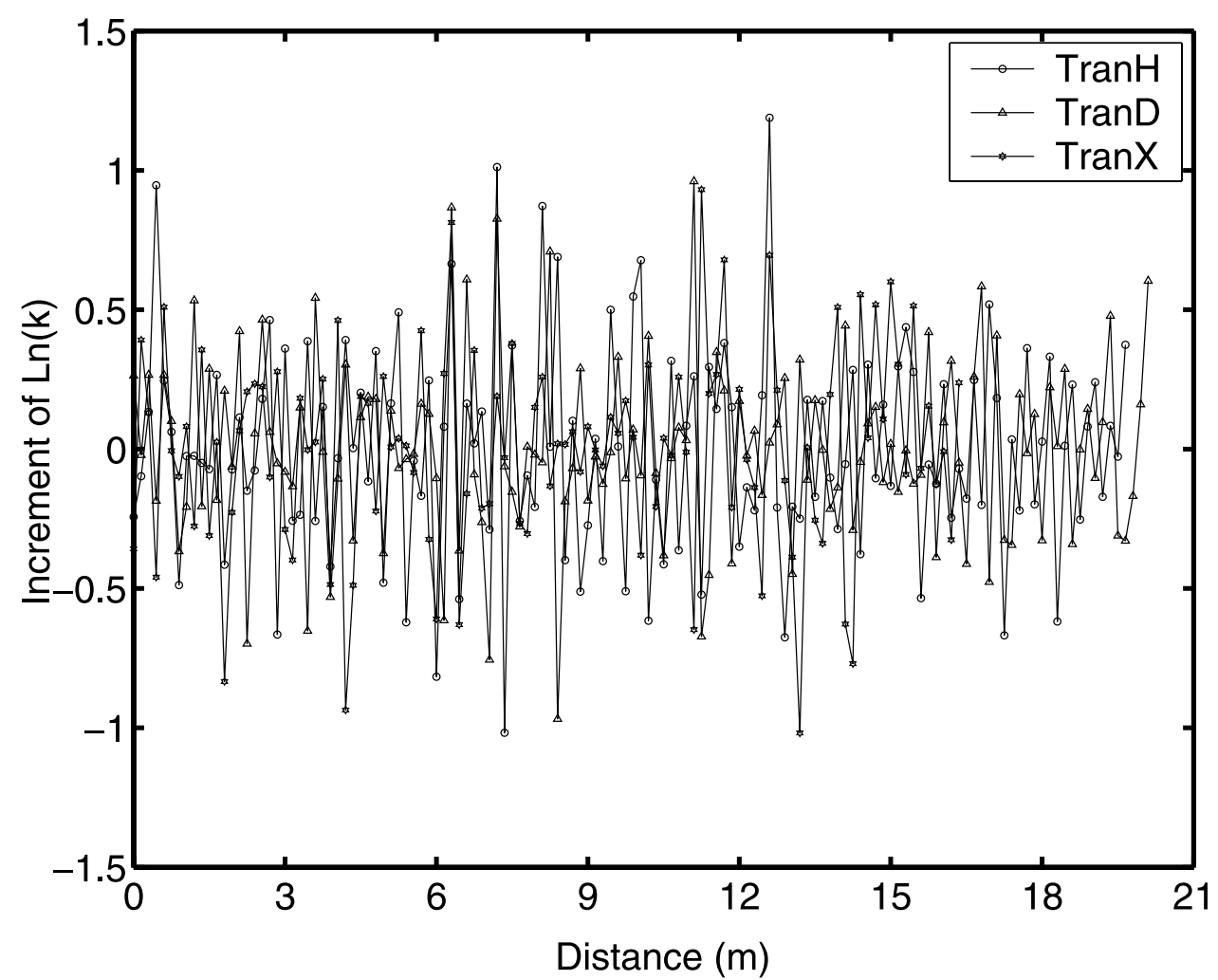

Figure 3. Plots of Ln (k) increments as a function of position for data from the three transects of Figure 2. These plots appear to be a good approximation of a stationary stochastic process. 


\section{HETEROGENEOUS SEDIMENT PROPERTIES AND TURBULENCE}

have been studied for several decades, especially since the well-known stochastic theory developed by Kolmogorov in the 1940s (Frisch, 1995), but in this application, the corresponding non-stationary process in an Eulerian Sense is not evident.

Stochastic fractals had their mathematical origin in the study of non-stationary stochastic processes with stationary increments. For this case, the focus falls naturally on the increments of a property, the difference between the property values measured at two points a known distance apart, rather than simply the property value itself at each point. Thus, if a property is measured at $\mathrm{n}$ points with a constant separation, $h$, one will have $n-1$ increments associated with $h$, also called the lag. These increment distributions may then be studied using statistical techniques. For example, an obvious first step would be to attempt to fit a probability density function (PDF) or a cumulative distribution function (CDF) to the increment distributions. Such distributions would be expected to vary in some way with the lag size, which is one way to define quantitatively the "scale" associated with a set of local measurements. Since irregular functions appear irregular on all scales of measurement, mathematical representations must reproduce this property, with the possibility of doing so in some orderly manner that (hopefully) agrees with experiment. It turns out that mathematicians were working on such representations throughout the first half of the $20^{\text {th }}$ century, with that early work collected in a series of classical texts by William Feller.

As discussed in Feller [1971], the most fundamental property of PDF's capable of representing increment distributions in an orderly manner is that such distributions be infinitely divisible. A probability distribution, $\mathrm{PD}$, is said to be infinitely divisible if and only if for any number, $n$, it can be represented as the sum of $\mathrm{n}$ independent random variables with a common distribution. Mathematically, this may be written as:

$$
P D=P D_{1, n}+P D_{2, n}+\ldots \ldots+P D_{n, n}
$$

All so-called stable distributions, which include the Lévystable distributions and the Gaussian special case, are infinitely divisible [Feller, 1971]. Thus it was natural for these distributions to be proposed as candidate PDFs for $\ln (\mathrm{K})$ increments. The important aspects of stable distributions as PDF's for property increments of a given lag will be introduced using the Gaussian probability density function $(\operatorname{GPDF}(\mathrm{x})$ ). (For a more general development that includes the Lévy-stable case see Molz et al., 2004.) For a mean of zero and a variance $\sigma^{2}$, the Gaussian distribution is given by:

$$
G P D F(x)=\frac{1}{\sigma \sqrt{2 \pi}} \exp \left(-\frac{x^{2}}{2 \sigma^{2}}\right)
$$

The fundamental property of the Gaussian distribution upon which infinite divisibility is based may be stated as follows: the PDF of $\mathrm{n}$ independent Gaussian random variables is still a Gaussian variable with a variance given by:

$$
\sigma^{2}=\sum_{i=1}^{n} \sigma_{i}^{2}
$$

where $\sigma$ is the standard deviation of the $i^{\text {th }}$ independent random variable.

In order to make Equation (3) more concrete, let us note specifically that we are dealing with the increments of a property, $P$, over a lag, $h, \equiv \Delta_{h} P$. Then the variance of the increments for a given $h$ is $\left\langle\left(\Delta_{h} P\right)^{2}\right\rangle$, where the brackets denote the expected value. In order to change from a set of increments of lag, $h$, to a set of lag, $2 h$, one simply adds each neighboring pair of increments together, that is, for each $i$ : $P_{i+2}-P_{i}=\left(P_{i+2}-\mathrm{P}_{i+1}\right)+\left(\mathrm{P}_{i+1}-\mathrm{P}_{i}\right)$. Combining this with (3) yields the scaling relationship:

$$
\begin{aligned}
<\left(\Delta_{2 h} P\right)^{2}> & =<\left[\left(\Delta_{h} P\right)+\left(\Delta_{h} P\right)\right]^{2}> \\
& =<\left(\Delta_{h} P\right)^{2}>+<\left(\Delta_{h} P\right)^{2}> \\
& =2<\left(\Delta_{h} P\right)^{2}>,
\end{aligned}
$$

and for a change from lag, $h$, to an arbitrary lag of, $r h$, (4) obviously generalizes to:

$$
<\left(\Delta_{r h} P\right)^{2}>=\mathrm{r}<\left(\Delta_{h} P\right)^{2}>
$$

Note that this scaling relationship may be extended to statistical moments of order $k$, in which case we would have:

$$
<\left(\Delta_{r h} P\right)^{k}>=r^{k / 2}<\left(\Delta_{h} P\right)^{k}>.
$$

As written, the scaling relationship implied by Equation (5) applies to Gaussian distributions with independent (uncorrelated) increments, which are the increments of Brownian motion. In 1968, Mandelbrot and Van-Ness extended Equation (5) to include the case of correlated increments. The result is:

$$
\begin{gathered}
<\left(\Delta_{r h} P\right)^{2}>=r^{2 H}<\left(\Delta_{h} P\right)^{2}>, \\
\text { with } 0<H<1 .
\end{gathered}
$$

This generalization is consistent with the existence of longrange positive correlation $(H>1 / 2)$ or negative correlation $(H<1 / 2)$ in the property increments, with the classical case of independent increments given by $H=1 / 2$. $H$ is called the Hurst coefficient, and when correlation is present, the increments constitute fractional Gaussian noise. The non-stationary sums of the increments are known as fractional Brownian motion. Convenient abbreviations are $\mathrm{fGn}$, and $\mathrm{fBm}$. To extend Equation (3) to include the Lévy-stable PDF, the exponent " 2 " is simply replaced by the Lévy stable index " $\alpha$ " (in which case the $\sigma_{i}$ 's are scale parameters, and not the standard deviations, which do not exist in this case). The resulting 
stochastic processes, analogous in several senses to fGn and $\mathrm{fBm}$, are called fractional Lévy noise (fLn) and fractional Lévy motion (fLm). However, the infinite variance feature of the Lévy-stable PDF makes this distribution a poor candidate for the present applications [Painter, 2001; Lu and Molz, 2001]. The properties and applications of both Gaussian and Lévy-stable fractals in subsurface hydrology have been reviewed recently [Molz et al., 2004].

\subsection{Results of Field Studies}

In order to determine if the stochastic fractal theory described in the introduction actually applies to field $\ln (\mathrm{K})$ data, it is necessary to compare field measurements with theoretical predictions. For Gaussian fractals, two main predictions apply: 1) the increments of $\ln (\mathrm{K})\left(\Delta_{h} \ln (\mathrm{K})\right)$ should be distributed normally for each $h$ value with mean zero and variance $\sigma_{h}^{2}$, and 2) the variance scaling relation given by Equation (7) should be satisfied by the measured variances. Most studies reported during the past decade have supported a variance scaling relationship. However, for smaller scale measurements, such as those obtained with a bore-hole flowmeter, the situation shown in Figure 4 was common [Meerschaert et al., 2004] For smaller lags, the increment distributions displayed a distinctly non-Gaussian shape, with peaking around the mean, and PDF tails that decayed in an approximately exponential manner. These attributes were also documented by Painter [2001], who presented PDF tails that decayed slower than the Lévy-stable case but faster than the Gaussian case (and in that sense anticipated the present work). For larger lags, Gaussian behavior appears. It was the observed peaking around the mean that led in part to the initial suggestion that the Lévystable PDF family should be considered as candidate distributions for the $\Delta_{h} \ln (\mathrm{K})$. However, the infinite variance feature could not be accommodated, and some of the larger data sets, such as the MADE data set (Figure 4), showed convergence to the Gaussian PDF as h increased, a property that was inconsistent with the Lévy-stable model [Liu and Molz, 1997; Meerschaert et al., 2004]. By mid-2003, the present authors also realized that similar variation was being displayed by velocity increment distributions and other properties measured in turbulent flows [Meerschaert et al., 2004]. Here, more data are available over a larger range of $h$, and increment PDFs roughly display the behavior $\operatorname{PDF}(\mathrm{x}) \sim \exp \left(-c x^{\beta}\right)$, with $c$ and $\beta$ constants and $x=$ property increment of interest over the lag $h$. As $h$ increases from smaller to larger values in steady turbulent flows, or if $h$ is held constant and the turbulent intensity is decreased from larger values to smaller values, $\beta$ is observed to increase from values $<1$ (sometimes called stretched Laplace behavior), through approximately unity (pure Laplace or double exponential behavior) and ultimately approaches 2 (Gaussian behavior). This type of behavior is illustrated in
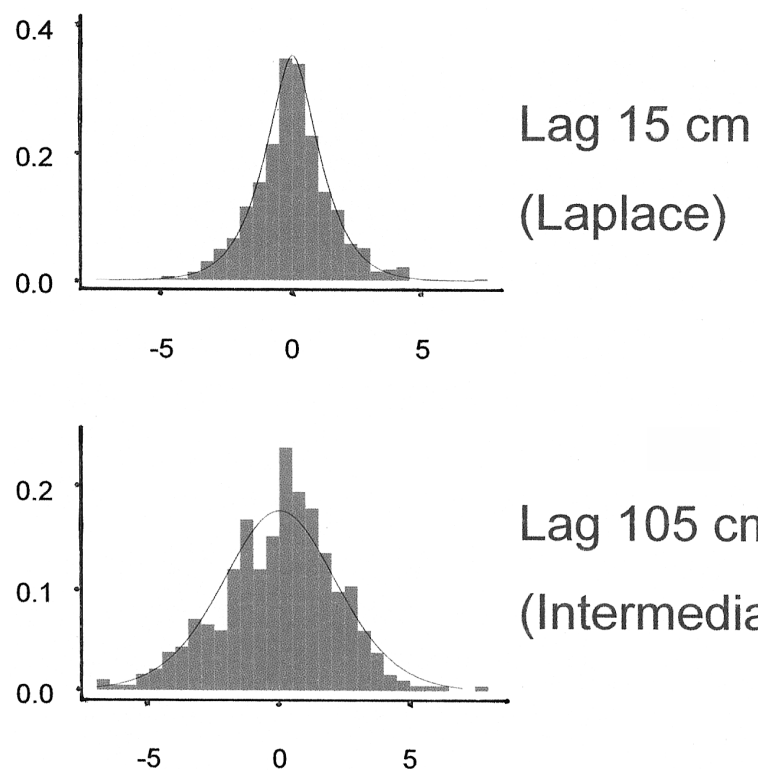

Lag $105 \mathrm{~cm}$

(Intermediate)

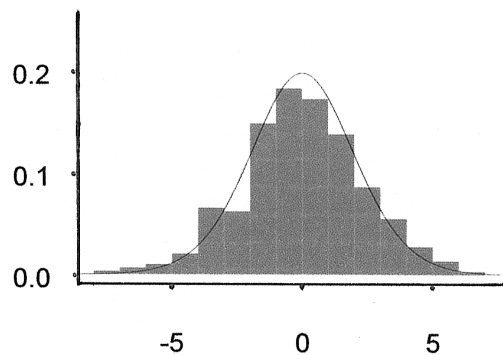

Lag $135 \mathrm{~cm}$

(Gaussian)

Figure 4. Frequency distributions of the $\ln (\mathrm{k})$ increments measured at the MADE site using a borehole flow-meter for lags of 15, 105 and $135 \mathrm{~cm}$. The distribution for the $15 \mathrm{~cm}$ lag is nearly pure Laplace, while that for a lag of $135 \mathrm{~cm}$ is approaching a Gaussian distribution.

Figure 5, which contains plots of simulated temperature increment distributions in turbulent flow, with the turbulent intensity increasing and $h$ fixed [Ching and Tu, 1994]. Experimental studies of velocity increment distributions have shown similar qualitative scaling properties [van de Water, 1998]. The possibility of some connection between turbulence and permeability distributions in sediments, which are commonly deposited in turbulent fluid flow fields, is interesting and should be investigated further.

\section{A NEW STOCHASTIC FRACTAL}

\subsection{Desired Properties}

Based on the experimental work discussed above, a stochastic fractal able to model heterogeneity in $\ln (\mathrm{K})$ should have the following attributes: (1) The process should be spatially (or temporally) non-stationary with stationary 


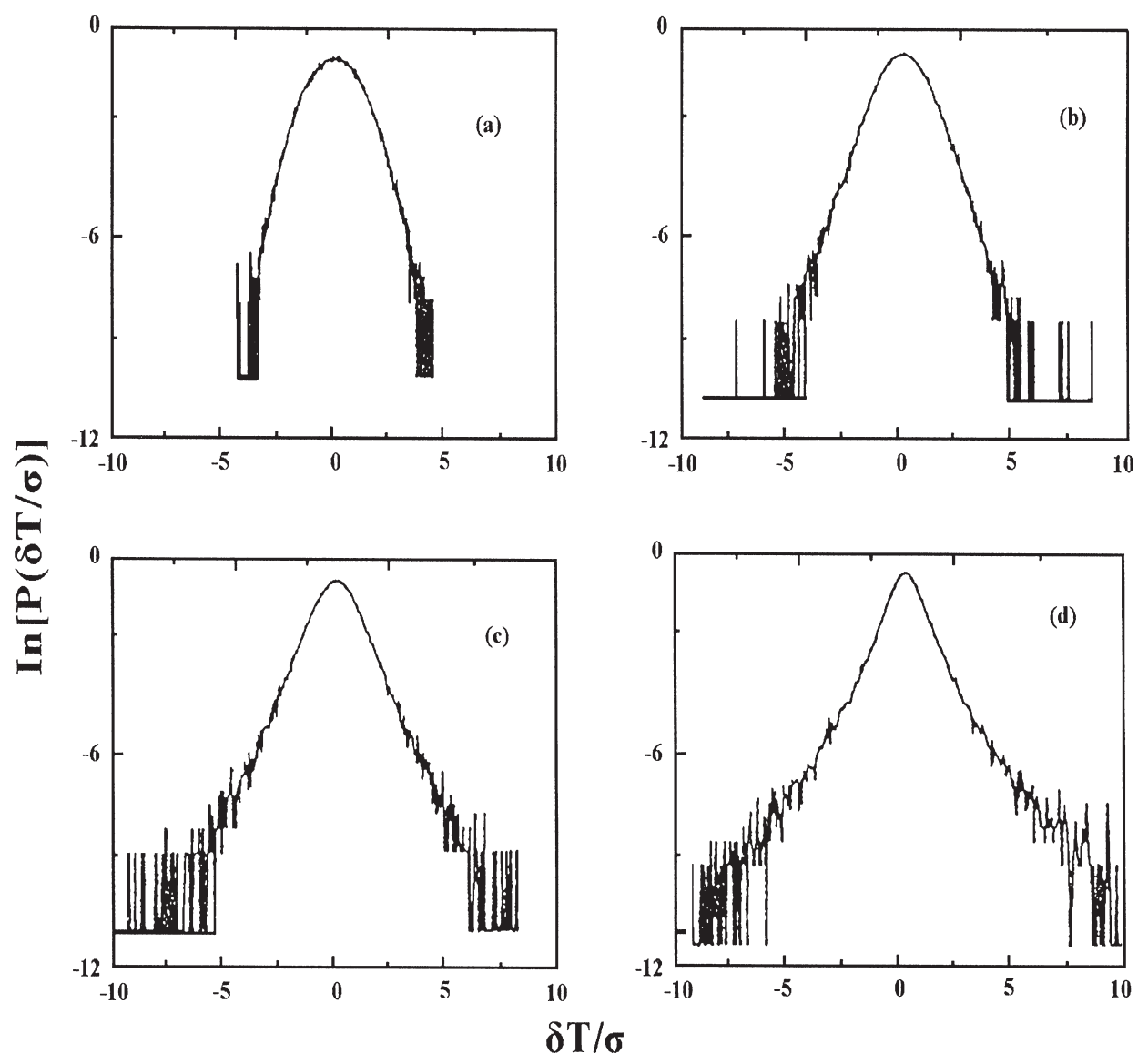

Figure 5. Semilog plots of frequency distributions for simulated temperature fluctuations in turbulent flows of varying intensity. In these simulations, the lag remained constant while the turbulent intensity increased from (a) through (d). Frequency distributions changed from near Gaussian (a), through Laplace (b and c) to the so-called stretched Laplace (d) [After Ching and Tu, 1994].

increments; (2) Statistical moments should remain bounded; and (3) Decay of the increment distribution tails should vary from being slower than exponential through exponential and on to a Gaussian decay as the lag size increases. Can such a stochastic process be identified?

Although not widely realized, the Laplace PDF is infinitely divisible [Kotz et al., 2001], and therefore can form the basis for what appears to be a new type of stochastic fractal. Some basic attributes of this stochastic process were presented by Meerschaert et al. [2004]. The stationary increment process was called fractional Laplace noise (fLan), and the non-stationary sum of increments was called fractional Laplace motion (fLam).

\subsection{Development of fLam and fLan}

Since the Laplace distribution is infinitely divisible, one can define a stochastic process $\{X(t), t \geq 0\}$ with stationary (homogeneous), independent increments that starts at the origin and such that the lag $v$ increment, $\Delta_{v} X(t)=X(t+v)-X(t)$, has a Laplace distribution with scale parameter $\sigma$ (Kotz et al., 2001). Lag $s$ increments of the resulting Laplace motion that have characteristic functions (ch.f.) of the form

$$
\phi_{s}(u)=1+\left(\sigma^{2} u^{2} / 2\right)^{-s / v}, u \in R,
$$

are known as generalized Laplace laws, and their densities (see [12] below) can be written in terms of the modified Bessel functions of the third kind (Kotz et al., 2001). When $s=v$ the above reduces to the Laplace ch.f., so the parameter $v>0$, determines the "Laplace scale". This process can be obtained as a Brownian motion $\left\{B_{H}(t), t \geq 0\right\} \quad(H=1 / 2)$ subordinated to the gamma process,

$$
\{X(t), t \geq 0\}={ }^{d}\left\{B_{H}\left(\Gamma_{t}\right), t \geq 0\right\} .
$$

The gamma process $\left\{\Gamma_{t}, t \geq 0\right\}$ is a Lévy process that starts at the origin, has independent and homogeneous increments, and the distribution of the lag $s$ increment has a gamma 
distribution with shape parameter $\alpha=s / v$ and scale $\beta=1$, denoted by $\mathrm{G}(\alpha, \beta)$, whose PDF is

$$
f(x)=\left[\beta^{\alpha} \Gamma(\alpha)\right]^{-1} x^{\alpha-1} e^{-x / \beta}, x>0 .
$$

Consequently, the marginal distributions of Laplace motion are scale mixtures of normal distributions of the form

$$
X(t)={ }^{d}\left(\sigma^{2} G t^{2 H}\right)^{1 / 2} Z,
$$

where $H=1 / 2$, the variable $Z$ is standard normal, and $G_{t}$ has the gamma distribution $G(t / v, 1)$. Written in Bessel function form, the PDF corresponding to logs increments of the uncorrelated Laplace family is given by

$$
g_{s}(t)=\frac{\sqrt{2}}{\sqrt{\pi} \sigma \Gamma(s / v)}\left(\frac{|t / \sigma|}{\sqrt{2}}\right)^{s / v-1 / 2} K_{s / v-1 / 2}(\sqrt{2}|t / \sigma|)
$$

where $K$ is the modified Bessel function of the $3^{\text {rd }}$ kind of index $s / n-1 / 2$. For a fixed value of $v$, different values of $s$ correspond to different members of the family. Several family members are plotted in Figure 6.

As developed so far, $X(t)$ is a stochastic process with uncorrelated increments. As such, it is analogous to Brownian motion, so it is desirable to try to extend the process to be analogous to the autocorrelated process fractional Brownian

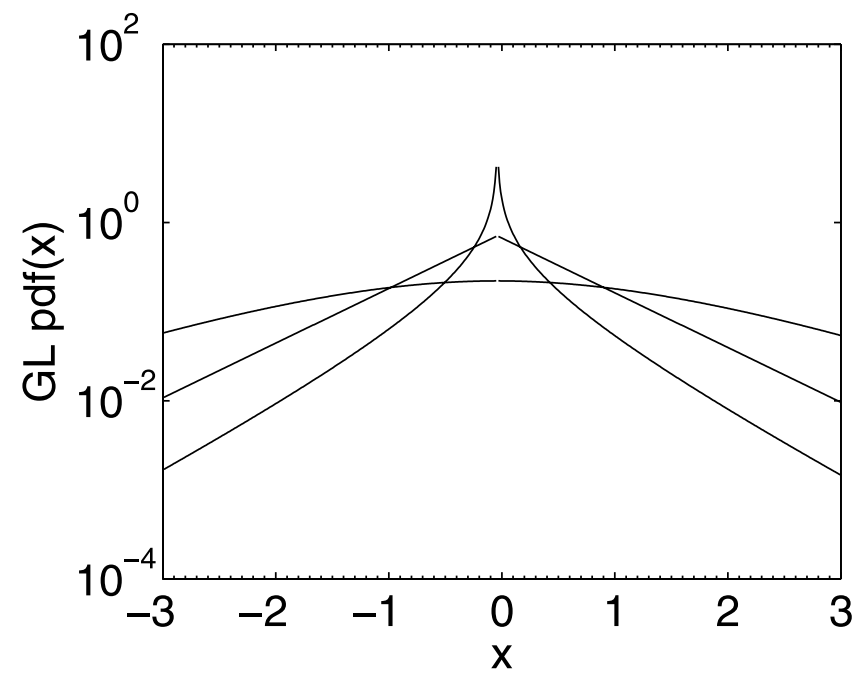

Figure 6. Semi-log plots of selected members of the generalized Laplace family, based on Equation (12), with zero mean and $v=1$. The concave upward (sharply peaked) curve $(s=0.25)$ is below what we call the Laplace scale, the two straight lines $(s=1)$ represent a classical Laplace PDF (double exponential), and the concave downward curve $(s=4)$ represents a generalized family member above the Laplace scale and moving towards a Gaussian distribution $(s=\infty)$. motion $(\mathrm{fBm})$. Therefore, starting with Laplace motion, Meerschaert et al. [2004] defined a process with correlated increments by subordinating a $\mathrm{fBm}$ with Hurst parameter $H$ $(0 \leq H \leq 1)$ to the gamma process via (9). FBm is a centered Gaussian process defined by the covariance function

$$
\begin{aligned}
& <B_{H}(t) B_{H}(s)>=\frac{\sigma^{2}}{2}\left(|t|^{2 H}+|s|^{2 H}-|t-s|^{2 H}\right) \\
& t, s \geq 0,
\end{aligned}
$$

where $\sigma$ is the standard deviation of $B_{H}(1)$. Following Meerschaert et al. (2004), we call the resulting process the fractional Laplace motion (fLam). The marginal distributions of this process are still of the form (11), but this time the stochastic variance $G_{t}^{2 H}$ has the generalized gamma distribution with PDF of the form

$$
\begin{aligned}
& h(x)=[2 H \Gamma(t / v)]^{-1} x^{t /(2 v H)-1} \exp \left(-x^{1 /(2 H)}\right) \\
& x>0
\end{aligned}
$$

(It is a power of a gamma variable.) The covariance structure of the fLam is easy to derive from that of a $\mathrm{fBm}$ (given by (13)) via conditioning on the values of the gamma process, leading to

$$
\begin{aligned}
& <X(t) X(s)>= \\
& \frac{\sigma^{2}}{2}\left(\frac{\Gamma(2 H+t / v)}{\Gamma(t / v)}+\frac{\Gamma(2 H+s / v)}{\Gamma(s / v)}-\frac{\Gamma(2 H+|s-t| / v)}{\Gamma(|s-t| / v)}\right) \\
& t, s>0 .
\end{aligned}
$$

By using the asymptotics of the gamma function (Stirling's formula), one can show that when $t, s$, and $|s-t|$ get large, (15) converges to the covariance function of $\mathrm{fBm}$. Lag $h$ increments of fLam $Y(k)=X(h k+h)-X(h k), k=0,1,2, \ldots$ form a stationary sequence called a fractional Laplace noise (fLan) with parameters $\sigma, v, h>0$ and $0<H<1$ - see Meerschaert et al. (2004). By stationarity, the marginal distribution of $Y(k)$ is the same as that of $X(h)$. The covariance function of this process, $r(n)=\langle Y(k) Y(k+n)>$, can be derived from that of $X(t)$, leading to

$$
\begin{aligned}
& r(n)=\frac{\sigma^{2}}{2}\left(\frac{\Gamma(2 H+(n+1) h / v)}{\Gamma((n+1) h / v)}\right) \\
& +\frac{\sigma^{2}}{2}\left(\frac{\Gamma(2 H+(n-1) h / v)}{\Gamma((n-1) h / v)}-2 \frac{\Gamma(2 H+n h / v)}{\Gamma(n h / v)}\right) \\
& n \geq 2
\end{aligned}
$$

Using representation (11) in conjunction with moments of the generalized gamma and normal distributions, one obtains 
the following expression for the $p^{\text {th }}$ (absolute) moment of a fLam $X(t)$ :

$$
S_{p}(t)=\left\langle|X(t)|^{p}>=\sigma^{p} \sqrt{\frac{2^{p}}{\pi}} \Gamma\left(\frac{p+1}{2}\right) \frac{\Gamma(H p+t / v)}{\Gamma(t / v)} .\right.
$$

This behaves like a power law, $S_{p}(t) \sim C_{p} t \zeta(p)$, for small and large values of $t$.

\subsection{FLan and fLam Simulations With Uncorrelated Increments}

Using results developed in Kotz et al. [2001], it is straightforward to construct the generalized Laplace family describing distributions of uncorrelated increments. The general structure of the uncorrelated, generalized, symmetric Laplace family with mean zero and $v=1$ is shown in Figure 6. This figure is based on plots of selected members from Equation (12). Additional plots may be found in Figure 4.2 of Kotz et al. [2001]. One may observe qualitatively that the type of behavior being observed in subsurface heterogeneity and turbulence (Figures 4 and 5, respectively) is displayed by the various members of the generalized Laplace family, with the case of $s$ $<1$ being analogous to what some individuals have been calling "stretched Laplace" [Ching and Tu, 1994]. Shown in Figures 7 and 8 are realizations of fLan and fLam, again for the case of uncorrelated increments. The noise process shown in Figure 7 was generated by simulating independent and identically distributed realizations of the generalized (symmetric) Laplace distribution, with $s=0.01$, utilizing the density

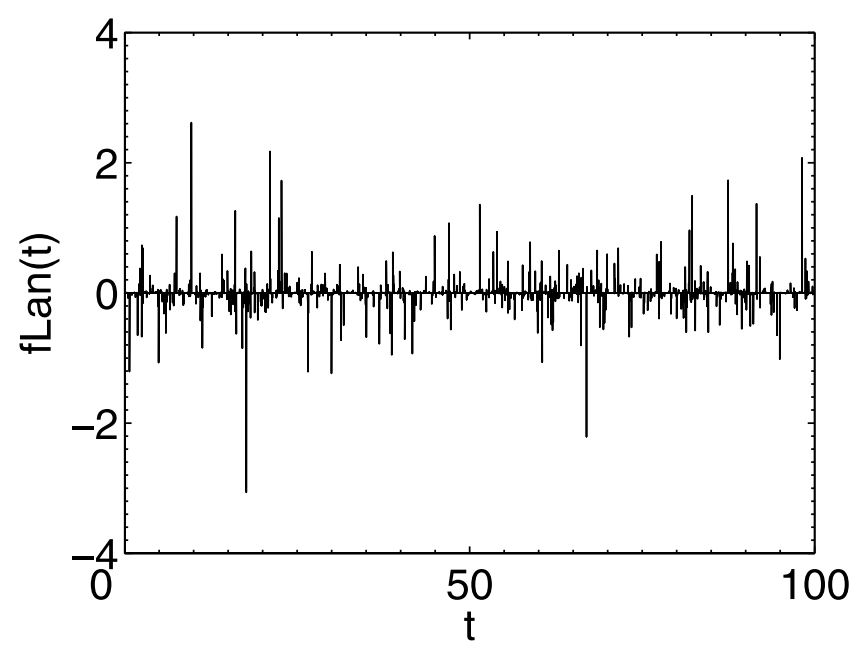

Figure 7. A realization of fractional Laplace noise for the case of uncorrelated increments. Such a process could potentially represent $\ln (\mathrm{k})$ increments, in which case " $\mathrm{t}$ " would represent distance along a transect, similar to the plot in Figure 3. Alternatively, fLan might represent turbulent velocity increments measured at a fixed point as a function of time.

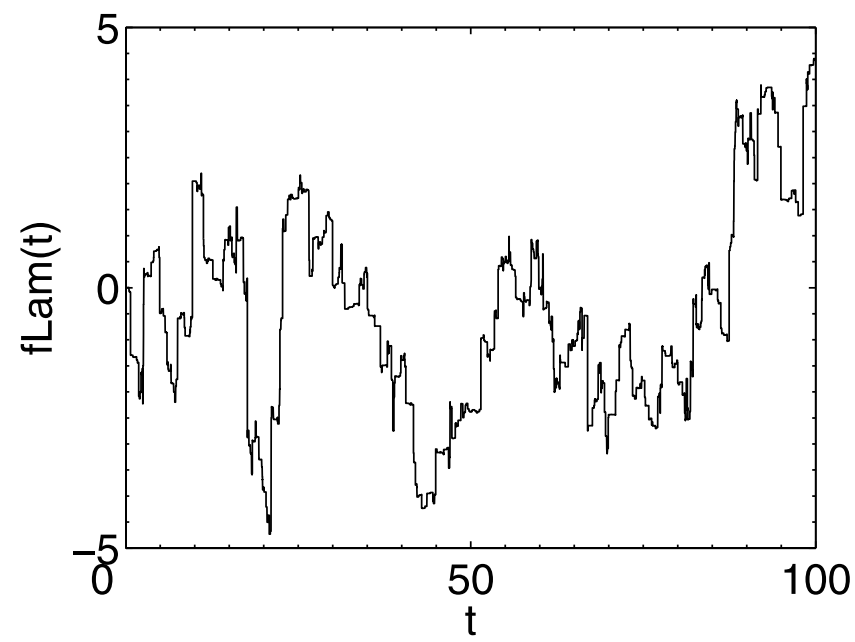

Figure 8. A realization of fractional Laplace motion that is the sum of the increments shown in Figure 7 above. Because the underlying increment PDFs change form markedly with lag, this stochastic process looks very different on different scales of observation. In this sense, it is more versatile than fBm or fLm.

function given by Kotz et al. [2001]. Taking a cumulative sum of the noise process gives the motion displayed in Figure 8.

What we see at the scale of the plots is a stochastic process that appears more like a Lévy flight than a Brownian motion. However, the noise (increment) process has finite statistical moments of all orders [Kotz et al., 2001], and will appear Gaussian at sufficiently large lags. The intermittency of the process is due mainly to input from the increment PDFs below the Laplace scale where the distributions peak highly around zero and have tails that decay slower than $e^{-x}$.

\section{DISCUSSION}

The fundamental difference between fLam/fLan and other similar stochastic processes is summed up in Figure 6. In the somewhat analogous Gaussian and Levy-stable processes, increment PDFs for all lags will be either Gaussian or Lévystable. In the new stochastic process, however, increment PDFs are highly variable with lag, especially for lags below the value of $s / v=1$, which yields the Laplace scale when $H=0.5$. Below the $s=v$ lag, the PDFs develop increasingly higher peaks around the mean of zero, and more slowly decaying tails. This favors fluctuations that cluster around the zero value but exhibit occasional large changes, which is characteristic of intermittent behavior. As s increases above $v$ however, behavior becomes much more regular as the increment PDFs approach a Gaussian shape. Turbulent velocity and $\ln (\mathrm{k})$ data both display this type of variation with lag.

It is intriguing to see at least similar qualitative behavior displayed by fluctuations of several quantities in turbulence 
and fluctuations (increments) of $\ln (\mathrm{k})$ in heterogeneous sediments. (We have verified that fLan reproduces Kolmogorov's laws within the inertial range of turbulence, which corresponds to the Gaussian limit [Frisch, 1995].) Superficial reasoning would suggest at least the possibility of such a commonality, since many sediments are formed through turbulent processes involving mainly wind and water. However, there is a little-appreciated central limit theorem, called the geometric central limit theorem (GCLT), that applies to the Laplace PDF [Kotz et al., 2001; Meerschaert et al., 2004]. A physical analog of the GCLT could then possibly illuminate a connection between the generalized Laplace family and the physical measurements discussed in this chapter. It is beyond the scope of the present communication to discuss what such a potential connection might be. However, an outline of the GCLT is given below.

The GCLT is associated with the idea of a random geometric sum, which in turn involves the well-known geometric PDF. Such PDFs arise naturally in several physical contexts. Herein we will use the context of a waiting time distribution. Start with a sequence of time intervals and number them sequentially. If an event has a constant probability " $q$ " of occurring during any time interval, then the random number $(N)$ of the interval in which the event is observed for the first time has the geometric PDF given by:

$$
P(N=n)=P(T=n \Delta t)=q(1-q)^{n-1} ; n=1,2,3, \ldots
$$

where $P=$ probability, $n=$ event number, and $T$ is the waiting time for the event to occur. It is easy to see how (18) arises by observing that $(1-q)^{n-1}$ is the probability that the event will not occur in the first $n-1$ trials and $q$ is the probability that it will occur in the nth trial. Given the geometric PDF, the GCLT may be stated as follows:

1) Start with a set of independent, identically distributed and symmetric random variables (RVs).

2) Number the random variables in a consecutive manner $\left(R V_{j}, j=1,2,3, \ldots\right)$.

3) For each $i=1,2,3, \ldots$ choose $N_{\mathrm{i}}$ to be a geometric random variable (17) where $q=q_{i}$ converges to zero (so that the mean of $N_{i}$ converges to infinity).

4) For each i, form the sum: $Y_{i}=\sqrt{q_{i}} \sum_{j=1}^{N_{i}} R V_{j}$ where $Y_{i}$ $(i=1,2,3, \ldots)$ is the random number resulting from each sampling.

5) As $i$ approaches infinity (and $q_{i}$ converges to zero) the frequency distribution of the $Y_{i}$ will approach a Laplace PDF [Kotz et al., 2001].

We offer the GCLT as a basis for future study of possible connections between the generalized Laplace family and observed turbulent and heterogeneous phenomena.
The increased understanding associated with the full development of such a possible connection could have practical implications for improved simulation of flow and transport in natural systems. For example, it might improve capabilities by providing the basis for a better understanding of parameter scale-dependence that is a concern in the application of numerical models. Alternatively, we might develop a basis for understanding that there are fundamental limitations to our predictive capabilities when faced with pervasive natural heterogeneity. A mathematical basis for limited predictability is formulated within the modern theory of deterministic chaos, and fractal-like structures seem to be associated with chaotic processes [Faybishenko, 2004].

\section{CONCLUSIONS}

Data have suggested that the "order" inherent in many irregular processes in sedimentation (permeability, electrical resistivity) and turbulence (turbulent velocity and temperature, among others) may be found in the increment PDFs of selected measurements. In the past, fractional Gaussian noise and fractional Lévy-stable noise have been used as models for such increment distributions. However, careful analysis of measurements has indicated that the increment PDFs are neither Gaussian nor Lévy-stable over the range of measurement scales utilized. In particular, Lévy-stable tail behavior is not observed, and distinctly non-Gaussian PDFs are observed for smaller lags. As an alternative, we are suggesting a new type of fractal based on members of the generalized Laplace distribution. In analogy with standard terminology, the stationary increment process is called fractional Laplace noise, and summing the increments yields the corresponding non-stationary process called fractional Laplace motion. The uncorrelated increment cases would simply be called Laplace noise and Laplace motion, which would be analogous to Gaussian noise and Brownian motion. However, the analogy should not be carried too far, because the Laplace-based fractal is fundamentally different from Gaussian and Lévy stable fractals; in particular, it does not exhibit self-similarity or self-affinity [Kotz et al., 2001]. This is because the underlying increment PDFs, while all being members of the generalized Laplace family, change dramatically with scale, something that does not occur with Gaussian or Lévy stable fractals. Also, fLam/fLan does not fall completely within the class of multifractals that have been of much interest lately [Kozubowski et al., 2005].

At the level of analysis performed so far, we have been able to see some potential statistical commonality between fluctuation processes in both heterogeneous sediments and in turbulent flows, suggesting that there may be some underlying connection between these phenomena. Future research should be devoted to studying this possibility and also to developing the complete features of the generalized Laplace 
family. In physical applications, it may be that the geometric central limit theorem will play an important role in connecting the abstract mathematics to various types of measurements.

\section{REFERENCES}

Castle, J.W., F.J. Molz, S. Lu, and C.L. Dinwiddie, Sedimentology and facies-dependent permeability, John Henry member, straight cliffs formation (upper cretaceous), Utah, U.S.A., J. Sed. Research, 74, 270-284, 2004.

Ching, E.S.C., and Y. Tu, Passive scalar fluctuations with and without a mean gradient: A numerical study, Phys. Rev. E, 49, 1278-1282, 1994.

Faybishenko, B., Nonlinear dynamics in flow through unsaturated porous media: Status and perspectives, Rev. Geophys., 42, RG2003, 2004.

Feller, W., An Introduction to Probability Theory and its Applications, Vols. 1 and 2, John Wiley, New York, 1971.

Freeze, R.A., A stochastic-conceptual analysis of one-dimensional groundwater flow in non-uniform, homogeneous media, Water Resour. Res., 11, 725-741, 1975.

Frisch, U., Turbulence, Cambridge University Press, Cambridge, 1995.

Gelhar, L.W., and C.L. Axness, Three-dimensional stochastic analysis of macrodispersion in aquifers, Water Resour. Res., 19, 161-180, 1983.

Kotz, S., Kozubowski, and K. Podgórski, The Laplace Distribution and Generalizations, Birkhauser Boston, Cambridge, Mass, 2001.

Kozubowski, T.J., M.M. Meerschaert, and K. Podgórski, Fractional Laplace motion, Preprint No. 65, Department of Mathematics, University of Nevada, Reno, in review, 2005.
Liu, H.H., and F.J. Molz, Comment on "Evidence for non-Gaussian scaling behavior in heterogeneous sedimentary formations" by Scott Painter, Water Resour. Res., 33, 907-908, 1997.

$\mathrm{Lu}, \mathrm{S}$., and F.J. Molz, How well are hydraulic conductivity variations approximated by additive stable processes, Adv. Environ. Res., 5, 39-45, 2001.

Mandelbrot, B.B., and J.W. van Ness, Fractional Brownian motions, fractional noises, and applications, SIAM Rev., 10, 422-437, 1968.

Meerschaert, M.M., T.J. Kozubowski, F.J. Molz, and S. Lu, Fractional Laplace model for hydraulic conductivity, Geophys. Res. Lett., 31, L08501, 2004.

Molz, F.J., H. Rajaram, and S. Lu, Stochastic fractal-based models in subsurface hydrology: Origins, applications, limitations and future research questions, Rev. Geophys., 42, RG1002, 2004.

Painter, S., Flexible scaling model for use in random field simulation of hydraulic conductivity, Water Resour. Res., 37, 1155-1163, 2001.

van de Water, W.V.D., Statistics of longitudinal and transverse velocity increments, in: U. Frisch (ed.), Advances in Turbulence VII, Kluer Academic, Boston, 1998.

Paul D. Hyden, Mathematical Sciences, Clemson University, Clemson, South Carolina 29634.

Tom, J. Kozubowski, Mathematics and Statistics, University of Nevada, Reno, Nevada 89557.

Mark M. Meerschaert, Mathematics and Statistics, University of Otago, Dunedin, New Zealand.

Fred J. Molz, Environmental Engineering and Geology, Clemson University, 342 Computer Court, Anderson, South Carolina 29625. 


\section{Queries}

Q1. Kindly check and advise in Figure 1 caption, we inserted closing bracket.

Q2. Kindly check and advise in Equation no. 8 we inserted open bracket. 